\title{
Prognostic and Molecular Classification Value of Ki- 67 Index in Patients With Breast Cancer
}

Chang-song Wang ( $\nabla$ wangtmmu150@163.com )

No. 989th Hospital

\section{Junling An}

Henan Institute of Science and Technology

Tian Yun

NO. 989th Hospital

Xiaoyue Wang

Luoyang Normal University

\section{Lei Ren}

Luoyang First Hospital

Nian-long Meng

NO. 989th Hospital

Yan-sha Cao

NO. 989th Hospital

Xue-xia Lv

NO. 989th Hospital

Fu-lin Li

NO. 989th Hospital

\section{Research}

Keywords: Ki-67, breast cancer, survive, molecular type, prognosis

Posted Date: June 23rd, 2020

DOI: https://doi.org/10.21203/rs.3.rs-37462/v1

License: (c) (1) This work is licensed under a Creative Commons Attribution 4.0 International License.

Read Full License 


\section{Abstract}

Background: The Ki-67, which is a proliferative index, became more important in making treatment decisions of breast cancers. But a few factors limited its use in clinical practices, especially the assessment of positive cells percentage of Ki-67 and the cut-off value of Ki-67. In this study, we examined the expression of Ki- 67 by immunohistochemical method, and evaluated the value of Ki- 67 index in patients with breast cancers systematically.

Methods: The expression of Ki-67 was detected by immunohistochemical method, the relationship between over-survive (OS), disease free survive (DFS) of patients with BCs and Ki-67 index冈and the value of Ki-67 in molecular classification of BC were analyzed with SPSS ver. 18.0 and GraphPad Prism software.

Results: There were markedly difference between OS in patients with $\mathrm{BC}$ when the Ki-67 index arranged from $46 \%$ to $68 \%(\chi 2=6.87, P=0.0088 ; \chi 2=7.69, P=0.0056$ respectively), and the same results were also found when the positive density was added to the Ki-67 index, but the positive density has limited value in assessment the value of Ki-67. There were markedly difference between DFS in BCs when the Ki67 index arranged from $50 \%$ to $58 \%(\chi 2=7.31, P=0.0068 ; \chi 2=7.88, P=0.005)$. When using $14 \%$ as a cut-off point to classify the molecular type of $\mathrm{BC}$, the Luminal A-type patients had statistical differences with patients with HER-2 over-expression, with triple negative type BC in term of OS respectively $(\chi 2=4.8$, $P=0.028 ; \chi 2=3.89, P=0.049)$. There's statistical difference between OS of patients with HER-2 overexpression BC when the Ki-67 index fell in the range of $49 \%-60 \%(\chi 2=4.97, P=0.0259 ; \chi 2=5.61, P=$ 0.0178 , respectively). There were statistical differences between the Luminal A-type BC and HER-2 overexpression $B C$ in term of OS $(X 2=4.65, P=0.031)$ according to suggestions of the CSCO consensus in 2019, there were statistical differences between two groups of Luminal B HER-2 (-) type BCs when the Ki67 index was $52 \%(\chi 2=6.61, P=0.0101)$.

Conclusions: $\mathrm{Ki}-67$ index can be used to assess the OS and DFS of patients with $\mathrm{BC}$, and the positive density of $\mathrm{Ki}-67$ has little value in assessment of prognosis in patients with BC. Different molecular classification methods may influence the assessment of prognosis and the results of molecular type in patients with BC.

\section{Background}

According to the latest national statistical data on cancer of China National Cancer Center in 2019, the incidence rate of breast cancer (BC) of Chinese was 45.29/100,000 in China. BC ranks the second among all cancers in term of incidence rate and the top position in term of incidence rate of cancers in women. Breast cancer is the fifth cancer in view of death rate among all cancers in women, which amounts to about 70,000 cases / year. Ki-67 is a key marker in prognosis and molecular classification of BC. In 2011, a consensus was reached on the St Gallen Conference, which required to using $14 \%$ as the critical value of Ki-67 to distinguish Luminal A-type and Luminal B-type BCs [1, 2]. Some guidelines also provided 
ambiguous expressions of critical value of Ki-67 as "high expression" and "low expression". Some experts suggested to using $\geq 20 \%$ as the critical value of "high expression" state of Ki-67 [3,4]. The CSCO Guideline in 2019 suggested that the critical value of Ki-67 shall be determined according to practical situations of various laboratories. Most Chinese experts agreed that $<15 \%$ is low expression and $>30 \%$ is high expression. When $\mathrm{Ki}-67$ index is $15 \%-30 \%$, it is suggested to make clinical decisions according to secondary pathology consultation or other indexes [5].

In practical pathological diagnosis, the cut-off point of $\mathrm{Ki}-67$ is difficult to be determined and has poor reproductivity. Different laboratories, technicians with different degree of familiarity and pathological diagnosis doctors with or without training might get different results. In particular, it is more difficult to have standard operation after the cutoff point of Ki-67 is solidified. Moreover, whether staining intensity of Ki-67 is meaningful to prognosis and molecular classification is a controversial difficulty at present. This study focuses on how to judge percentage of positive cells of Ki-67 simply and conveniently and whether there's a certain interval for the convenience of operation of clinical pathologists to improve repeatability.

\section{Methods}

\section{Clinical data}

Paraffin samples of 288 patients with BC from January, 2005 to July, 2013 in NO. $989^{\text {th }}$ Hospital was collected for a retrospective study. Among these patients, there were 4 males $(1.39 \%)$ and 284 females (98.61\%). All respondents were aged between 25 and 84, averaging at 48 years old. Specifically, there were 2 cases younger than 30 years old, 230 patients between 30-60 years old, and 56 patients over 60 years old. With respect to lymphatic metastasis, there were 154 cases without lymphatic metastasis (N0), 66 cases with 1-3 nodes of lymphatic metastasis (N1), 39 cases with 4-9 nodes of lymphatic metastasis (N2), and 20 cases with $\geqq 10$ nodes of lymphatic metastasis (N3). According to pathological stages of AJCC (pTNM), there were 8 cases in Stage 0, 42 cases in Stage IA, 104 cases in Stage IIA, 60 cases in Stage IIB, 43 cases in Stage IIIA, 1 case in Stage IIIB, 20 cases in Stage IIIC, and 1 case in Stage IV (Tab.1). The follow-up time lasted for 2 - 157 months, averaging at 82 months. In addition, three cases had metastatic lesions and 6 cases had not undergone radical operation. None patient had received chemoradiotherapy before the operation. Samples were fixed with $10 \%$ neutral formalin and embedded with paraffin. All patients had complete follow-up data. The inclusion standards of respondents were as follows: 1) all patients were diagnosed through breast puncture or postoperative examination after radical surgery. 2) None patients received chemotherapy or radiotherapy before operation. 3) All patients had complete follow-up data. 4) Patients died for breast cancer metastasis or relapse. Patients who met one of following conditions were excluded: 1) without complete follow-up data, 2) received thermotherapy or radiotherapy before operation, 3) patients died for reasons other than breast cancer metastasis or relapse, 4) patients with poor living quality caused by other reasons or patients died for other tumors. This study was approved by the ethics committee of NO. $989^{\text {th }}$ hospital and all included respondents signed the informed consent. 


\section{Immunohistochemical assay of Ki-67 in BC samples}

Tumor samples were fixed in 10\% paraformaldehyde solution buffered with neutral phosphate-buffered

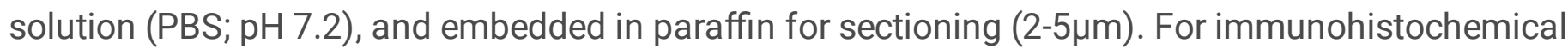
analyses on Benchmark XT system (Ventana, AZ, USA), deparaffinized sections were immersed in $0.3 \%$ $\mathrm{H}_{2} \mathrm{O}_{2}$ in methanol for 30 min to eliminate endogenous peroxidase activity, followed by incubation with PBS containing $1 \%$ normal serum corresponding to the secondary IgG and $1 \%$ bovine serum albumin (BSA) to reduce nonspecific reactions. The sections were incubated with rabbit anti-human Ki-67 (MIB-1) (dilution 1:200; Maxim, Fuzhou, China). After incubation with biotinylated secondary Abs at room temperature (RT) for 1 hour, immune complexes were amplified with Streptavidin-Alkaline Phosphatase (SAP) immunohistochemical staining kits (Maxim, Fuzhou) following the manufacturer's instructions. Negative control was done by omitting the primary antibodies and substituting with corresponding normal isotype-matched immunoglobulin IgG.

\section{Results analysis}

Ki-67 protein positive lies on cell nucleus. It is judged positive for occurrence of yellow nuclei or brown particles. The whole section was previewed under a low power lens before interpretation to judge whether the staining is uniform. If there's even staining, samples were observed under a high power lens (40x) to count more than 500 cancer cells. If there's uneven staining, the hot spot region with concentrated positive cell number was chosen to count more than 500 cancer cells. Samples which approached to cutoff point were judged again by 2 another independent pathologists and the average Ki-67 index was calculated. Finally, the mean value was chosen as the final Ki-67 index value. Samples with staining intensity unable to be determined and ambiguous cell profiles were excluded from counting. Besides, staining intensity of tumor cells was scored: light yellow ( 1 score), deep yellow ( 2 scores) and brown ( 3 scores). Staining index $=$ percentage of positive cellsx staining intensity. The minimum and maximum staining indexes were $1 \%$ and $285 \%$, respectively.

\section{Molecular classification}

Molecular classification was performed by the consensus on the St Gallen Conference in 2011 (Luminal A-type and Luminal B-type BCs were distinguished by using $14 \%$ as the critical value of Ki-67) and suggestions of CSCO guideline in 2019 (If critical value of Ki-67 is smaller than $<15 \%$, it is low expression. If critical value of $\mathrm{Ki}-67$ is higher than $30 \%$, it is high expression) respectively. When expressions of ER (+), HER-2 (-) and Ki- 67 are between 15\% - 30\%, molecular classification of BC was performed according to percentage of positive expression cells of PR. Under this circumstance, $P R=20 \%$ was used as the critical value according to suggestions of CSCO in 2019. If $P R>20 \%$, samples were determined as the Luminal Atype $B C$. If $P R<20 \%$, samples were determined as the Luminal B-type BC.

\section{Statistical analysis}


The statistical analyses were completed by using SPSS ver. 18.0 (SPSS Inc., Chicago, IL). Survival data (overall survival, OS and disease-free survival, DFS) were analyzed by using Kaplan-Meier survival curves and compared using log-rank tests among different groups. Statistical significance was 2-sided, and Pvalues $<0.05$.

\section{Results}

\section{Clinical-pathological characteristics of patients with BC}

Among the patients with BC enrolled in this study, invasive ductal carcinoma not otherwise specified (DCS, NOS) was found in $90.97 \%$ of patients, ductal carcinoma in situ was found in $2.78 \%$, invasive lobular carcinoma (LCS) was found in $2.08 \%$, invasive micropapillary carcinoma was found in $1.04 \%$, mucinous carcinoma was found in $2.43 \%$, medullary carcinoma was found in $0.35 \%$, and apocrine carcinoma was found in $0.35 \%$. And then, there were $44.80 \%$ (125/279) patients with positive lymph node and $55.20 \%$ (154/279) without lymph nodes metastasis. According to the AJCC stage, $2.87 \%(8 / 279)$ of patients had stage $0,15.05 \%$ (42/279) of patients had stage I, 58.78 (164/279) of patients had stage II,

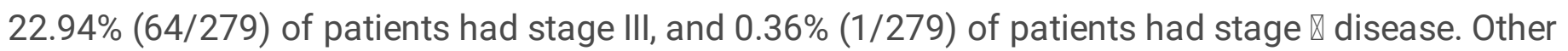
pathological characteristics of the patients are shown in Table 1. The median follow-up time was 82 months (range from 2 to 157 months).

\section{Relationship between $\mathrm{Ki}-67$ index and overall survival (OS) of patients with BC}

After staining and interpretation, there were 285 samples which could count more than 500 tumor cells. Therefore, the practical statistical group had $285 \mathrm{BC}$ samples. The median Ki-67 value was $60 \%$ (range from $1 \%$ to $95 \%$ ), and Ki-67 $\leq 1 \%$ accounted for $8.4 \%$ (24/285) of patients, Ki- $67 \geqq 90 \%$ accounted for $3.51 \%(10 / 285)$. According to statistical analysis, OS of patients with BC declined significantly when Ki67 index fell within $46 \%-68 \%$, showing statistical differences $(\chi 2=6.87, P=0.0088 ; \chi 2=7.69, P=$ 0.0056 respectively. Tab.2 and Fig. 1$)$. This indicated that BC patients with $\mathrm{Ki}-67$ index ranging between $46 \%-68 \%$ had poor prognosis. This interval deserved key attentions when interpreting Ki-67 index of patients with $\mathrm{BC}$.

Whether staining intensity of Ki-67 of tumor cells is significant to judging OS of patients with BC? Staining intensity was added based on Ki-67 index. Results showed that there were statistical differences of OS between two groups when the critical value was 133\% after accumulation of Ki-67 index and staining intensity and patients with high expression of Ki-67 index had relatively poorer prognosis $(\chi 2=$ 7.82, $P=0.0052$. Tab.3 and Fig.1C). However, there's no statistical difference of OS between the group with staining intensity and the group without staining intensity $(\chi 2=0.001, P=0.9780$, Tab.3). This reflected that staining intensity of Ki-67 was not valuable to OS prediction of patients with BC.

\section{Relationship between expression of $\mathrm{Ki}-67$ in $\mathrm{BC}$ and disease-free survival (DFS) of patients}


At the end of follow-up time, 36 patients among 288 patients with BC developed metastasis or in-situ relapse at different positions. According to statistical analysis, DFS of patients with BC declines gradually with the increase of $\mathrm{Ki}-67$ index from $50 \%$ to $58 \%(\chi 2=7.31, P=0.0068 ; \chi 2=7.88, P=0.005$ respectively). This revealed that Ki-67 index was conducive to judge DFS of patients with $B C$ when it was in the range of $50 \%-58 \%$ (Tab. 4 and Fig.2).

\section{Molecular classification of BC based on consensus of the St. Gallen Conference in 2011}

Among 288 patients with BC, molecular classification of 262 patients with invasive ductal carcinoma was performed by using the critical Ki67 index value (14\%) according to the consensus of the St. Gallen Conference in 2011. It distinguished 61 cases of Luminal A-type BC, 113 cases of Luminal B-type, 46 cases of HER-2 over-expression, and 42 cases of triple negative type BC. According to statistical analysis, Luminal A-type BC patients had statistical differences with patients with HER-2 over-expression and triple negative type $B C$ in term of OS $(\chi 2=4.80, P=0.0280 ; \chi 2=3.89, P=0.0490)$, (Tab.5 and Fig.3A, 3B).

To further investigate the relationship between Ki-67 index and each molecular-type of BC, a statistical analysis on the relationship between percentage of Ki-67 positive cells and molecular type of patients with BC was carried out. It found that there's statistical difference between OS of patients with HER-2 over-expression $\mathrm{BC}$ when $\mathrm{Ki}-67$ index fell in the range of $49 \%-60 \%(\chi 2=4.97, P=0.0259 ; \chi 2=5.61, P=$ 0.0178 , respectively) (Fig.3C, 3D), but there's no statistical difference among Luminal A-type group, Luminal B-type group and triple negative type group.

\section{Molecular classification of patients with $\mathrm{BC}$ based on molecular classification proposed by Chinese Society of Clinical Oncology (CSCO) in 2019}

Among 288 patients with BC, 258 patients with invasive ductal carcinoma adopted molecular classification according to standards in CSCO in 2019. If percentage of Ki-67 positive cells was lower than $15 \%$, it is determined as low expression. If percentage of Ki- 67 positive cells was higher than $30 \%$, it was determined as high expression. When percentage of Ki-67 positive cells fell in the interval of $15 \%$ $30 \%$, molecular classification of PC was performed according to state of HER-2 and percentage of PR positive cells. In other words, $\mathrm{PR}=20 \%$ was used as the critical value according to suggestions of the CSCO consensus in 2019 when expressions of HER-2 (-) and Ki-67 were in the range of $15 \%-30 \%$. If PR > $20 \%$, patients were determined as Luminal A-type BC; otherwise, patient were determined as Luminal Btype BC. According to molecular classification results, there were 91 cases of Luminal A-type BC, 79 Luminal B-type BC, 46 cases of HER-2 over-expression BC, and 42 cases of three-yin type BC. According to statistical analysis results, there were statistical differences between the Luminal A-type BC and HER-2 over-expression $\mathrm{BC}$ in term of OS $(\chi 2=4.65, P=0.0310)$. The patients with HER-2 over-expression $\mathrm{BC}$ presented higher death rate and poorer prognosis, but it presented no statistical difference with Luminal B-type BC and triple negative type BC in term of OS (Tab.6 and Fig.4A).

Relationship between $\mathrm{Ki}-67$ index and prognosis of patients with specific molecular classification based on CSCO in 2019 was analyzed. According to statistical analysis, there were statistical differences 
between two groups of Luminal B HER-2 (-) type when the critical value of Ki-67 index was $52 \%(X 2=6.61$, $P=0.0101)$, (Figure 4B). Moreover, there were statistical differences between two groups of HER-2 overexpression type when Ki-67 index fell in the critical interval of $47 \%-60 \%(\chi 2=4.97, P=0.0259 ; \chi 2=5.61, P=$ 0.0178 , respectively) (Fig. 4C, 4D.), but these two groups showed no statistical difference with other molecular subtypes. Otherwise, it is interested that all of 19 patients with Luminal A-type BC survived when $\mathrm{Ki}-67$ index was $\leq 4 \%$ (within the follow-up visit period)

\section{Discussion}

Effects of Ki-67 in BC mainly could be divided into two parts: 1) molecular classification: this is particularly necessary when distinguishing Luminal A-type BC and Luminal B-type BC, because the former one only needs endocrine-therapy, while the later one needs systematic chemotherapy based on endocrine-therapy to achieve good clinical effects [6]. Hence, it is very necessary to distinguish these two types of BC in clinics. 2) judgment of prognosis of patients: many studies have demonstrated that patients with higher Ki-67 index had poor prognosis and they were easier to suffer relapse [7,8]. Patients with higher Ki-67 index will gain better pathological complete responses (pCRs), without considerations to HR state, HER-2 state and triple negative type of BC [9]. Some study even believed that Ki-67 index was unrelated with prognosis of patients with BC. Moreover, the prognosis was poorer when the Ki-67 index was lower [10]. Some study has demonstrated that other clinical pathological features of patients shall be considered during judging influences of $\mathrm{Ki}-67$ index on patients with $\mathrm{BC}$, including histological grading, lymphatic metastasis, tumor volume, existence of vascular invasion, etc [11].

Therefore, Ki-67 has not been widely applied in clinics. In addition to differences among observers, some clinicians depend more on Ki-67 division between "high Ki-67 group" and "low Ki-67 group" in determining the treatment. However, the critical value of "low Ki-67 group" ranges between 0 and $28.6 \%[12,13]$, showing significant differences. Therefore, how to determine critical value of $\mathrm{Ki}-67$ index and values of $\mathrm{Ki}-$ 67 index in judgment of prognosis and molecular classification of patients with $B C$, and even how to make reasonable and efficiency use of Ki-67 in $\mathrm{BC}$ are of significant importance.

Immunohistochemical staining is the most common analytical method of Ki-67. This method is not only economic and simple, but also needs no complicated equipments. Immunohistochemical staining is easy to be promoted. However, Ki-67 has limited practical values in clinical practices, because there are many interference factors before, during and after experiment. Interferences before the experiment are mainly selection of stationary liquid, fixed time and cold ischemia time. Interferences during the experiment generally include type of antibody, antigen repair method, staining development time and proficiency of technicians. Interferences after the experiment mainly include counting technique of positive cells, professional training of observers, and accurate recognition of positive cells [14-17]. Influencing factors before and during the experiment can be avoided through standardized operation as much as possible, including choosing $10 \%$ neutral formalin as the fixed liquid, controlling time of samples in fixed liquid after separation within $30 \mathrm{~min}$, assuring at least $24 \mathrm{hrs}$ of fixed time, and using the primary antibody of the same batch from the same factory, the same antigen repair method after optimization as well as the 
same technician. Counting of positive cells after experiment was the most difficult work. To optimize the counting method and decrease differences among observers as much as possible, the International Ki-67 in Breast Cancer Working Group offered some suggestions to promote applications of Ki-67 in BC: counting at least 3 high power fields ( $\times 40$ objectives) or counting 500-1000 tumor cells, including invasion edges of tumor and hot spot region [18].

In this study, percentage of Ki-67 positive cells in 285 patients with BC was calculated and it found that there were statistical significant differences among patients with $B C$ in term of $O S$ when the critical value of Ki-67 index fell within the interval of $46 \%-68 \%$. In other words, death rate of patients increased gradually and the prognosis deteriorated with the increase of Ki-67 index. Since the interval of Ki-67 index is large, it is easy to be promoted in pathologists and shows relatively high repeatability. Staining intensity of Ki-67 was included into the evaluation index, which also can influence OS prediction of patients with BC. However, the group considering staining intensity and the group without consideration to staining intensity showed no statistical significant differences in OS prediction. This proved that staining intensity of Ki-67 has not significant values in OS prediction of patients with BC. Zhu pointed that that setting the critical value of $\mathrm{Ki}-67$ index as $30 \%$ is an independent prognosis factor of OS and DFS of three-yin cancer [19]. Patients with triple negative cancer and Ki-67 $>30 \%$ had relatively poorer prognosis, especially for patients in Stage I. When the critical value of Ki-67 index was $10 \%$, for both high expression ( $\geq 10 \%)$ and low expression ( $<10 \%)$, patients with BC show statistical differences in OS and recurrence-free survival (RFS) when there's low PR expression ( $<20 \%)$. In particular, patients with high Ki67 expression and low PR expression had the poorest prognosis [20]. Similarly, Ki-67 index is significant to DFS judgment of patients with BC. According to this study, DFS of patients with BC decreased gradually when $\mathrm{Ki}-67$ index value increased from $50 \%$ to $58 \%$, but the interval of $\mathrm{Ki}-67$ index to judgment of DFS was relatively small and special attentions shall be paid to it. However, some study show that when the critical value of Ki-67 index was determined 14\%, patients with Luminal-type BC and high Ki-67 expression ( $\geq 14 \%$ ) and low PR expression $(<50 \%)$ achieved a relatively high clinical complete remission (CR) from neoadjuvant chemotherapy [21].

There's a "gray zone" during molecular classification to patients with BC based on Ki-67 index, especially when different methods were applied. During molecular classification of patients with $\mathrm{BC}$ based on the consensus of St. Gallen Conference in 2011, it is relatively difficult to set $14 \%$ as the critical value of Ki-67 index in practical applications. In particular, the repeatability is poorer when the primary counting is between $12 \%-16 \%$. However, there are still counting errors by the same technician and the error is even larger by multiple technicians. In this study, the same observer was assigned to make three times of counting in 2 days for patients whose $\mathrm{Ki}-67$ index ranges between $10 \%$ and $20 \%$. Next, the mean value was used as the final Ki-67 index. This can decrease errors among different observers as much as possible and the total cell counting is increased significantly. The biggest defect lies in high workloads and it is only applicable to the situation with small sample sizes. During molecular classification based on CSCO standards in 2019, it needs repeated counting or decision-making according to other evidences when the Ki-67 index ranges between 15\% - 30\% [5], which absolutely increases workload and leads to 
poor repeatability as well as increased errors. How to decrease "grey zones" and differences in molecular classification of BC is the key and difficulty of this study.

During molecular classification of patients with BC based on consensus on St. Gallen Conference in 2011, the OS of patients with Luminal A-type BC shows no statistical difference with those of patients with HER-2 over-expression and patients with triple negative type BC. OS of patients with HER-2 overexpression BC show statistical difference when $\mathrm{Ki}-67$ index fell within $49 \%-60 \%$, but there's no statistical difference among other subtypes. During molecular classification based on consensus of CSCO in 2019, OS of patients with Luminal A-type BC shows statistical difference with that of HER-2 over-expression BC in term of OS, but it shows no statistical difference with other subtypes. Differences caused by two different molecule classification methods might influence prognosis judgment of patients. When Ki-67 index is $52 \%$, there's statistical difference of OS among patients with Luminal B HER-2 (-) subtypes. Moreover, there's statistical difference of OS among patients with HER-2 over-expression BC when Ki-67 index is $47 \%$. These further reflect differences of $\mathrm{Ki}-67$ index among different molecular classification methods, which deserve special attentions.

In a word, a systematic study on application values of $\mathrm{Ki}-67$ index to patients with $\mathrm{BC}$ is carried out. Results show that $\mathrm{Ki}-67$ index has a large value range to judge $\mathrm{OS}$ of patients with $\mathrm{BC}$ and it can decrease interpretation differences among different observers. Staining intensity can influence OS prediction based on Ki-67 index slightly. The interval of Ki-67 index for DFS judgment of patients with BC is relatively small and the $\mathrm{Ki}-67$ index proposes a high requirement on interpretation in practices. Different molecular classification methods can influence prognosis judgment and classification of patients with BC. Therefore, molecular classification methods shall be chosen carefully in order to provide a more effective clinical treatment. Moreover, other clinical pathological features shall be considered.

\section{Abbreviations}

BC: breast cancer; DFS: disease-free survival; CSCO: Chinese Society of Clinical Oncology; OS: overall survival; RT: room temperature; SAP: Streptavidin-Alkaline Phosphatase; ER: estrogen receptor; PR: progesterone receptor.

\section{Declarations}

Acknowledgements We would like to thank all the patients for their contribution in this study.

Authors' contributions All of authors made contributions to the acquisition, analysis, or interpretation of data. CS W and JLA were involved in drafting the manuscript and revising it critically for important intellectual content. CSW, TY and XYW were responsible for literature search and manuscript preparation. $\mathrm{RL}, \mathrm{NLM}$ and YSC participated in the discussion for histological diagnosis and manuscript preparation. XXL collected the clinical data and postoperative clinical follow-up of the patient. YSC and FLL participated in the microscopic analyses. All authors read and approved the final manuscript. 
Funding None.

Availability of data and materials None.

Ethics approval and consent to participate This study was approved by the Ethics Committee of NO. $989^{\text {th }}$ Hospital.

Consent for publication Consent was achieved from all patients or their relatives.

Competing interests All authors have declared no conflicts of interest.

Author details 1. Department of Pathology, 989 th Hospital of PLA, Luoyang, 471031, P.R. China. 2. Department of Pathology and Pathophysiology, Medical College, Henan University of Science and Technology, Luoyang , 471023, P.R. China. 3. School of Electronic Commerce, Luoyang Normal College, Luoyang, 471000, P.R. China. 4. Department of Pathology, Luoyang First People's Hospital, Luoyang, 471000, P.R. China.

\section{References}

1. Goldhirsch A, Wood WC, Coates AS, et al. Strategies for subtypes - dealing with the diversity of breast Cancer: highlights of the St. Gallen international expert consensus on the primary therapy of early breast Cancer 2011. Ann Oncol 2011; 22:1736-47.

2. Cheang MC, Chia SK, Voduc D, et al. Ki67 index, HER2 status, and prognosis of patients with luminal B breast cancer. J Natl Cancer Inst 2009;101:736-50

3. Maisonneuve $P$, Disalvatore $D$, Rotmensz $N$, et al. Proposed new clinicopathological surrogate definitions of luminal A and luminal B (HER2-negative) intrinsic breast cancer subtypes. Breast Cancer Res 2014; 16:R65.

4. Coates AS, Winer EP, Goldhirsch A, et al. Tailoring therapies-improving the management of early breast cancer: St Gallen international expert consensus on the primary therapy of early breast Cancer. Ann Oncol 2015; 26:1533-46.

5. Li JB, Jiang ZF. Update and interpretation of 2019 guideline of Chinese Society of Clinical Oncology (CSCO): breast cancer. Chin J Surg Oncol 2019,11(3):155-160.

6. Goldhirsch A, Winer EP, Coates AS, et al. Personalizing the treatment of women with early breast cancer: highlights of the St Gallen International Expert Consensus on the Primary Therapy of Early Breast Cancer 2013. Ann Oncol 2013; 24(9): 2206-2223.

7. Nishimura R, Osako T, Nishiyama Y, et al. Prognostic significance of Ki-67 index value at the primary breast tumor in recurrent breast cancer. Mol Clin Oncol 2014; 2(6):1062-8.

8. Pathmanathan N, Balleine RL, Jayasinghe UW, et al. The prognostic value of Ki67 in systemically untreated patients with nodenegative breast cancer. J Clin Pathol 2014; 67:222-8. 
9. Chen X, He C, Han D, et al. The Predictive value of Ki-67 before neoadjuvant chemotherapy for breast cancer: A Systematic Review and Meta-Analysis. Future Oncol 2017; 13(9):843-857.

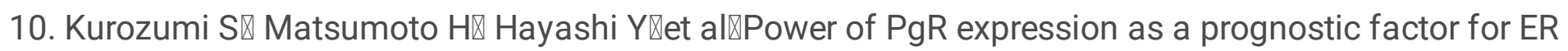
positive /HER-2 negative breast cancer patients at intermediate risk classified by the Ki67 labeling indexखBMC Cancer 2017; 17( 1): 354】

11. Kadivar M, Aram F. Assessment of Ki67 in Breast Cancer: A comparison between the Eye-10 method, stepwise counting strategy, and International System of Ki67 evaluation. Iran J Pathol 2020; 15(1): 13-18

12. Urruticoechea A, Smith IE, Dowsett M. Proliferation marker Ki-67 in early breast cancer. J Clin Oncol 2005; 23(28):7212-20.

13. Stuart-Harris R, Caldas C, Pinder SE, Pharoah P. Proliferation markers and survival in early breast cancer: a systematic review and meta-analysis of 85 studies in 32,825 patients. Breast 2008; 17(4):323-34.

14. Dowsett M, Nielsen TO, A'Hern R, et al. Assessment of Ki67 in breast cancer: recommendations from the International Ki67 in Breast Cancer Working Group. J Natl Cancer Inst 2011;103(22):1656-1664 .

15. McCormick D, Yu C, Hobbs C. The relevance of antibody concentration to the immunohistological quantification of cell proliferation-associated antigens. Histopathology 1993; 22(6):543-547.

16. Boon ME. Microwave-antigen retrieval: the importance of $\mathrm{pH}$ of the retrieval solution for MIB-1 staining. Eur J Morphol 1996; 34(5):375-379.

17. Polley MY, Leung SC, McShane LM, et al. An international Ki67 reproducibility study. J Natl Cancer Inst 2013;105:1897-906.

18. Fulawka L, Halon A. Ki-67 evaluation in breast cancer: the daily diagnostic practice. Indian J Pathol Microbiol 2017; 60(2):177-184.

19. Zhu X, Chen L, Huang B, et al. The prognostic and predictive potential of Ki-67 in triple-negative breast cancer. Sci Rep 2020; 10(1):225.

20. Kang YJ, Lee HB, Kim YG, et al. Ki-67 expression is a significant prognostic factor only when progesterone receptor expression is low in estrogen receptor-positive and HER2-negative early breast cancer. J Oncol 2019; 2019:7386734.

21. Silva LRD, Vargas RF, Shinzato JY, et al. Association of menopausal status, expression of progesterone receptor and $\mathrm{Ki} 67$ to the clinical response to neoadjuvant chemotherapy in Luminal breast cancer. Rev Bras Ginecol Obstet 2019; 41(2):710-717.

\section{Tables}


Tab.1 Pathological characteristics of 288 patients with $\mathrm{BC}$

\begin{tabular}{|c|c|c|}
\hline Factors & $\mathrm{N}$ & $\%$ \\
\hline \multicolumn{3}{|l|}{$\operatorname{Sex}(N=288)$} \\
\hline M & 4 & $1.39 \%$ \\
\hline FM & 284 & $98.61 \%$ \\
\hline \multicolumn{3}{|l|}{ Age (year) $(\mathrm{N}=288)$} \\
\hline$\leq 30$ & 2 & $0.69 \%$ \\
\hline $30-60$ & 230 & $79.86 \%$ \\
\hline$\nabla 60$ & 56 & $19.44 \%$ \\
\hline \multicolumn{3}{|l|}{ Histological type $(\mathrm{N}=288)$} \\
\hline DCIS & 8 & $2.78 \%$ \\
\hline DCS & 262 & $90.97 \%$ \\
\hline LCS & 6 & $2.08 \%$ \\
\hline invasive micropapillary carcinoma & 3 & $1.04 \%$ \\
\hline mucinous carcinoma & 7 & $2.43 \%$ \\
\hline medullary carcinoma & 1 & $0.35 \%$ \\
\hline apocrine carcinoma & 1 & $0.35 \%$ \\
\hline \multicolumn{3}{|l|}{ T stage $(\mathrm{N}=279)^{*}$} \\
\hline Tis & 8 & $2.87 \%$ \\
\hline T1b & 15 & $5.38 \%$ \\
\hline T1c & 49 & $17.56 \%$ \\
\hline T2 & 183 & $65.59 \%$ \\
\hline T3 & 22 & $7.89 \%$ \\
\hline T4 & 2 & $0.72 \%$ \\
\hline
\end{tabular}

$\mathrm{N}$ stage $(\mathrm{N}=279)$ *

\begin{tabular}{|lll|}
0 & 154 & $55.20 \%$ \\
N1 & 66 & $23.66 \%$ \\
N2 & 39 & $13.98 \%$ \\
\hline
\end{tabular}




\begin{tabular}{|lll|}
$\mathrm{N} 3$ & 20 & $7.17 \%$ \\
\hline AJCC stage $(\mathrm{N}=279)^{*}$ & & \\
\hline 0 & 8 & $2.87 \%$ \\
\hline$\triangle \mathrm{A}$ & 42 & $15.05 \%$ \\
\hline$\triangle \mathrm{A}$ & 104 & $37.28 \%$ \\
\hline$\triangle \mathrm{B}$ & 60 & $21.51 \%$ \\
\hline $\mathrm{A}$ & 43 & $15.41 \%$ \\
\hline$\square \mathrm{B}$ & 1 & $0.36 \%$ \\
\hline$\square \mathrm{C}$ & 20 & $7.17 \%$ \\
\hline$\square$ & 1 & $0.36 \%$ \\
\hline
\end{tabular}

Abbreviations: M, male; FM, female; DCS, NOS, invasive ductal carcinoma not otherwise specified; DCIS, ductal carcinoma in situ; LCS, invasive lobular carcinoma ; AJCC, American Joint Committee on Cancer.

* There were 3 cases had metastatic lesions and 6 cases had not undergone radical operation, there were no complete $\mathrm{T}$ stage, $\mathrm{N}$ stage and AJCC stage data.

Tab. 2 Relationship between Ki-67 index and OS of patients with $\mathrm{BC}$

\begin{tabular}{|lllll|}
\hline Ki-67 index & Death $(\mathrm{n})$ & Survive $(\mathrm{n})$ & $\chi 2$ & $P$ \\
\hline$<46 \%$ & 45 & 193 & 6.87 & 0.0088 \\
\hdashline $46 \%$ & 15 & 32 & & \\
\hline$<68 \%$ & 52 & 213 & 7.69 & 0.0056 \\
$\geqq 68 \%$ & 8 & 12 & & \\
\hline
\end{tabular}

Tab. 3 Relationship between staining intensity of Ki-67 and OS of patients with $\mathrm{BC}$

\begin{tabular}{|c|c|c|c|c|c|}
\hline Ki-67 index & Death $(n)$ & Survive (n) & Death rate (\%) & $x^{2}$ & $P$ \\
\hline$<46 \%$ & 45 & 193 & $18.91 \%$ & & \\
\hline$\geqq 46 \%$ & 15 & 32 & $31.91 \%$ & & \\
\hline$<133 \%$ & 46 & 196 & $19.01 \%$ & 0.001 & $0.9780 *$ \\
\hline$\geqq 133 \%$ & 14 & 29 & $32.56 \%$ & 7.82 & $0.0052^{*}$ \\
\hline
\end{tabular}


$* 46 \%$ vs $133 \%$ group; $*<133 \%$ vs $\geqq 133 \%$ group.

Tab. 4 Relationship between ki-67 index and DFS of patients with BC

\begin{tabular}{|lllll|}
\hline Ki-67 index & Death (n) & Survive $(\mathrm{n})$ & $\chi 2$ & $P$ \\
\hline$<50 \%$ & 34 & 181 & 7.31 & 0.0068 \\
$\geqq 50 \%$ & 11 & 26 & & \\
\hline$<58 \%$ & 35 & 185 & 7.88 & 0.0050 \\
$\geqq 58 \%$ & 10 & 22 & & \\
\hline
\end{tabular}

Tab. 5 Molecular type of patients with BC according to St. Gallen Conference in

\begin{tabular}{|llllll|}
\hline \multicolumn{7}{c|}{2011} \\
Molecular type & Death $(\mathrm{n})$ & Survive $(\mathrm{n})$ & Total $(\mathrm{n})$ & $\chi 2$ & $P$ \\
\hline Luminal A & 9 & 52 & 61 & & \\
\hline Luminal B HER-2 (-) & 13 & 58 & 71 & & \\
\hline Luminal B HER-2 (+) & 9 & 33 & 42 & & \\
\hline Over-expression HER-2 & 15 & 31 & 46 & 4.80 & $0.028^{*}$ \\
\hline Triple-negative & 13 & 29 & 42 & 3.89 & $0.049 *$ \\
\hline
\end{tabular}

*Luminal A type vs HER-2 over-expression type; * Luminal A type $v s$ triple negative type.

Tab. 6 Molecular type of patients with BC according to CSCO consensus in 2019

\begin{tabular}{|llll|}
\hline Molecular type & Death $(\mathrm{n})$ & Survive $(\mathrm{n})$ & Total $(\mathrm{n})$ \\
\hline Luminal A & 15 & 76 & 91 \\
\hline Luminal B HER-2 $(-)$ & 7 & 30 & 37 \\
\hline Luminal B HER-2 $(+)$ & 9 & 33 & 42 \\
\hline Over-expression HER-2 type* & 15 & 31 & 46 \\
\hline Triple-negative type & 13 & 29 & 42 \\
\hline
\end{tabular}

*Luminal A type vs HER-2 over-expression type $(\chi 2=4.65, P=0310)$. 
Figures
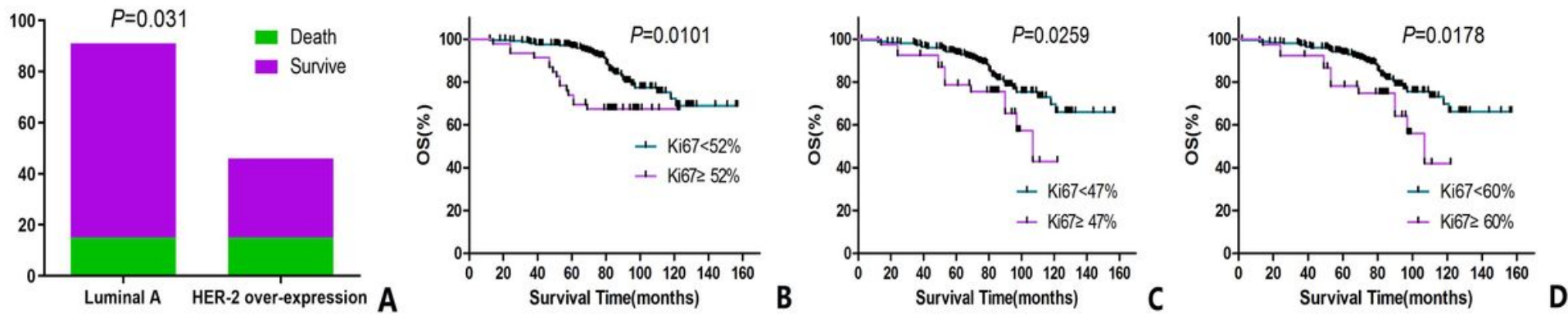

Figure 1

A: According to the 2019 CSCO meeting, the difference was seen between Luminal A type and overexpression of HER-2 type of BC. B: Luminal B HER-2 (-) type patients with Ki-67 labeling index ( $\geqq 52 \%)$ had significantly inferior OS rate compared with Ki-67 labeling index ( $<52 \%)$. C: the HER-2 over-expression patients with abeling index ( $\geqq 47 \%$ ) had significantly inferior OS rate compared with Ki-67 labeling index (<47\%). D: the HER-2 over-expression patients with Ki-67 labeling index ( $\geqq 60 \%)$ had significantly inferior OS rate compared with Ki-67 labeling index (<60\%).
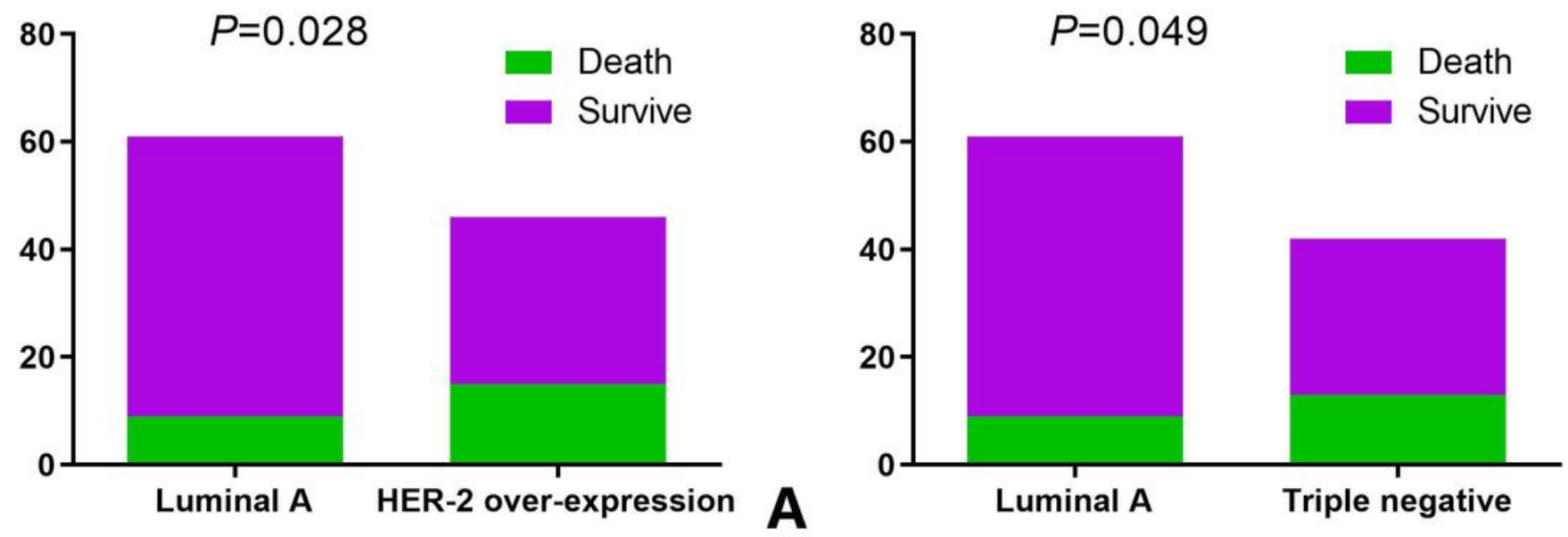

Luminal A

Triple negative

B
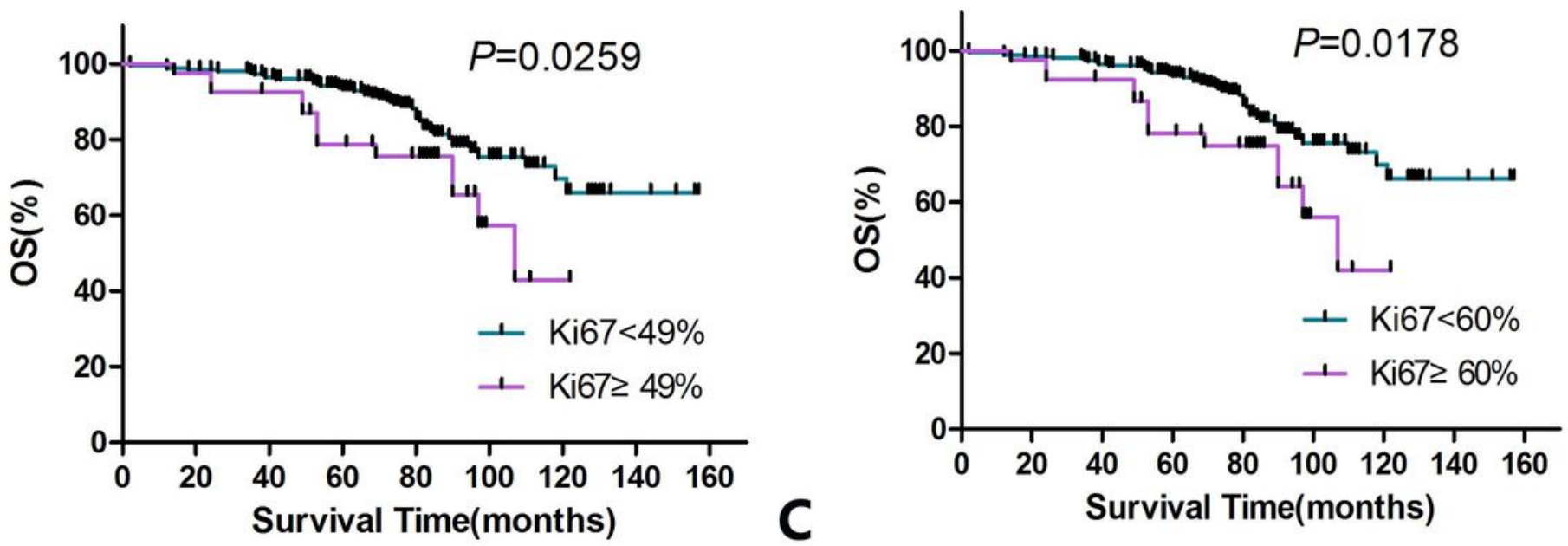
Figure 2

According to the 11th St. Gallen International Consensus Meeting, the difference was seen between Luminal A type and over-expression of HER-2 type, triple-negative type. A: Luminal A type vs overexpression of HER-2 type. B: Luminal A type vs triple-negative type. C: Patients with HER-2 overexpression BC with abeling index ( $\geqq 49 \%$ ) had significantly inferior OS rate compared with $\mathrm{Ki}-67$ labeling index (<49\%). D: Patients with HER-2 over-expression BC with Ki-67 labeling index ( $\geqq 60 \%)$ had significantly inferior OS rate compared with Ki-67 labeling index $(<60 \%)$.
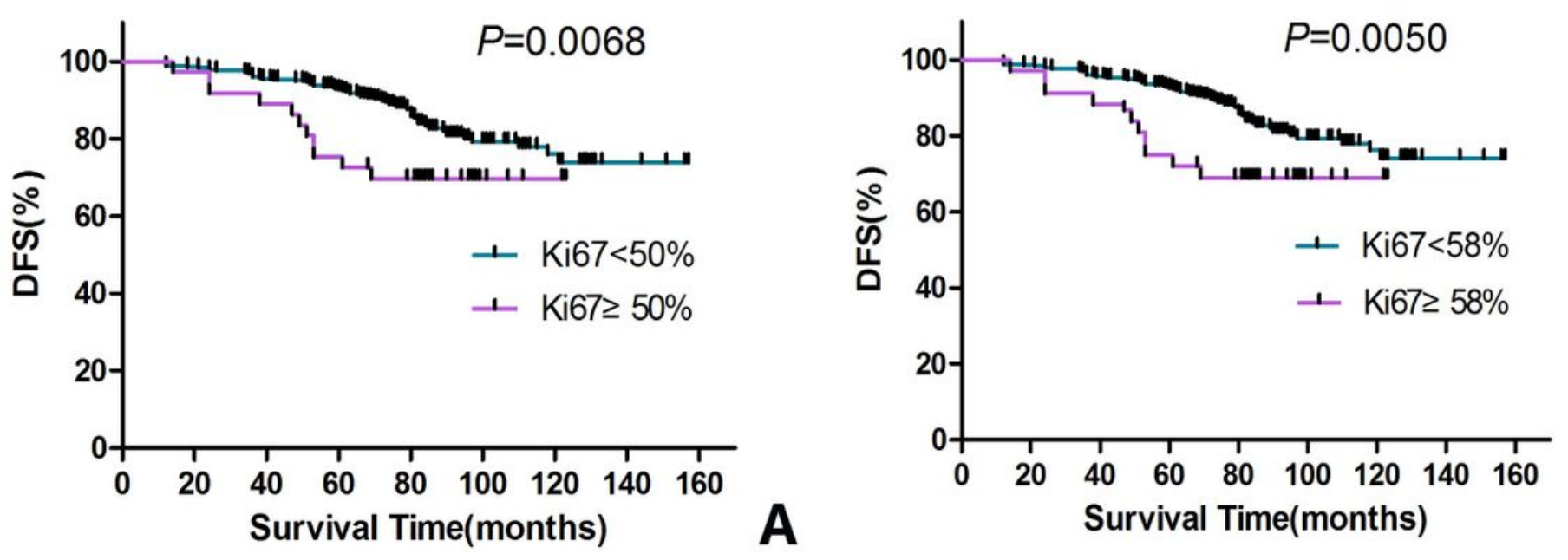

\section{Figure 3}

Kaplan-Meier curves (DFS) of the patients with BC according to the different Ki-67 cutoff point. A:

Patients with abeling index ( $\geqq 50 \%$ ) had significantly inferior DFS rate compared with Ki- 67 labeling index $(<50 \%)$. B: Patients with $\mathrm{Ki}-67$ labeling index ( $\geqq 58 \%$ ) had significantly inferior DFS rate compared with Ki67 labeling index $(<58 \%)$.
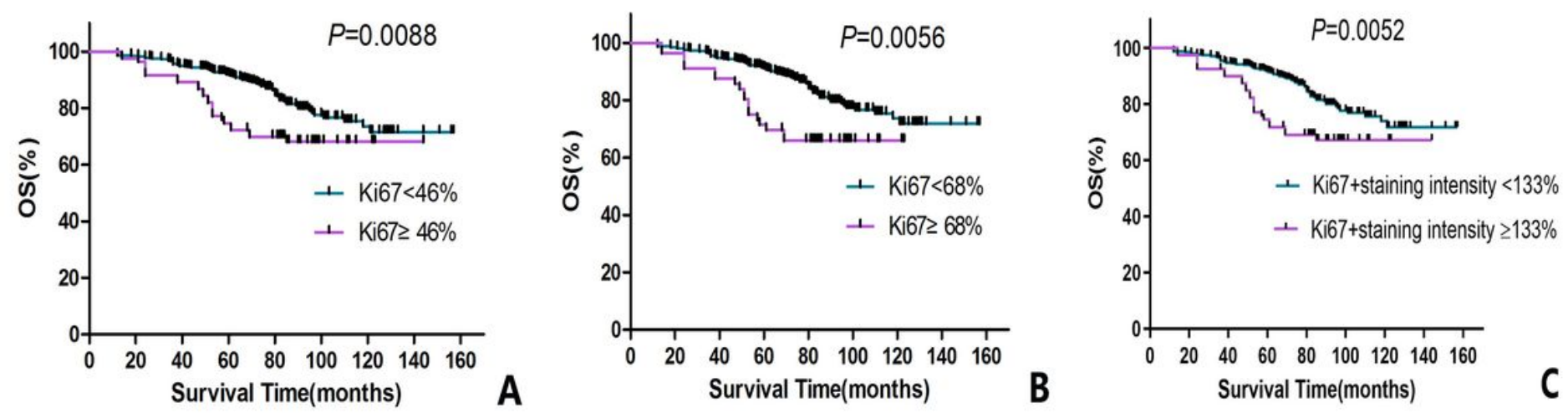

Figure 4 
Kaplan-Meier curves of the patients with BC according to the different Ki-67 index. A: Patients with Ki-67 labeling index ( $\geqq 46 \%$ ) had significantly inferior OS rate compared with Ki-67 labeling index (<46\%). B: Patients with Ki-67 labeling index ( $\geqq 68 \%$ ) had significantly inferior OS rate compared with Ki-67 labeling index (<68\%). C: Kaplan-Meier curves of the BC patients with Ki-67 labeling index plus different stained intensity. 